\title{
LONG SLIT ECHELLE SPECTROSCOPY OF SUPERNOVA REMNANTS IN M33
}

\author{
William P. Blair \\ The Johns Hopkins University, Baltimore, Md., U.S.A. \\ You-Hua Chu \\ The University of Illinois, Urbana, Il., U.S.A. \\ Robert C. Kennicutt \\ The University of Minnesota, Minneapolis, Mn., U.S.A.
}

\begin{abstract}
We have obtained long slit echelle spectroscopy for 10 of the brightest supernova remnants in M33 using the KPNO $4 \mathrm{~m}$ telescope. The profiles at $\mathrm{H} \alpha$ indicate bulk motions in the range $100-350 \mathrm{~km} \mathrm{~s}^{-1}$ in these remnants. Nearly all of the objects show signs of contamination by low velocity $\mathrm{H}$ II emission at some level. This affects the line intensities measured from low resolution data and may affect diameter measurements of these remnants.
\end{abstract}

\section{Introduction and Background}

Samples of supernova remnants (SNRs) in nearby galaxies are important for studies of SNR evolution and also provide probes of the galaxy in which they reside. The number of SNRs and their relative sizes can be related to the supernova rate and models of the interstellar medium. Relative emission line intensities can be used to estimate physical conditions and chemical abundances; a sample of remnants can then be used to investigate variations in abundances and abundance gradients in spiral galaxies.

The most effective method for finding SNRs in nearby galaxies has proven to be comparison of $[\mathrm{S} \mathrm{II}]$ and $\mathrm{H} \alpha$ imagery: SNRs have much higher $[\mathrm{S} \mathrm{II}] / \mathrm{H} \alpha$ ratios than $\mathrm{H}$ II regions and can often be identified even in somewhat confused regions of nearby galaxies. However, many SNRs are expected in giant H II regions, but confusion problems make application of the $[\mathrm{S} \mathrm{II}] / \mathrm{H} \alpha$ criterion impossible. We believe SNRs embedded in giant H II regions can be identified kinematically ( $c f$. Chu and Kennicutt 1986), but there are no integrated velocity profiles available for comparison.

The advent of more realistic shock model calculations (Cox and Raymond 1985; Dopita et al. 1984) makes it possible to interpret the relative line intensities of SNRs in terms of chemical abundances ( $c f$. Blair and Kirshner 1985). However, since the relative line intensities in H II regions and SNRs are different, any contamination of low resolution SNR spectra by $\mathrm{H}$ II emission can affect the abundance determinations. H II contamination might also cause problems with SNR diameter estimates, which are needed for SNR evolution studies.

These problems have motivated us to obtain echelle observations of SNRs in M33. At the scale of M33 ( 1 arcsec $\approx 3.5 \mathrm{pc}$ ), many of the brighter SNRs fall almost entirely in the slit, permitting measurements of integrated line profiles. Also, at high resolution and with a long slit, effects of $\mathrm{H} \mathrm{II} \mathrm{contamination} \mathrm{can} \mathrm{be} \mathrm{assessed.} \mathrm{Below} \mathrm{we} \mathrm{describe} \mathrm{these} \mathrm{observations}$ and some preliminary results.

\section{Observations}

We have used the echelle spectrograph in long slit mode on the $4 \mathrm{~m}$ telescope at Kitt Peak to obtain spectrograms of ten of the brightest SNRs in M33. A 79 line $\mathrm{mm}^{-1}$ grating and an interference filter were used to isolate the order containing both $\mathrm{H} \alpha$ and [N II] $\lambda \lambda 6548,6584$, while the long slit oriented E-W across the SNRs provided spatial information. The $800 \times 800$ TI- 4 chip was binned by 2 in the spatial direction (to decrease read-out noise) 


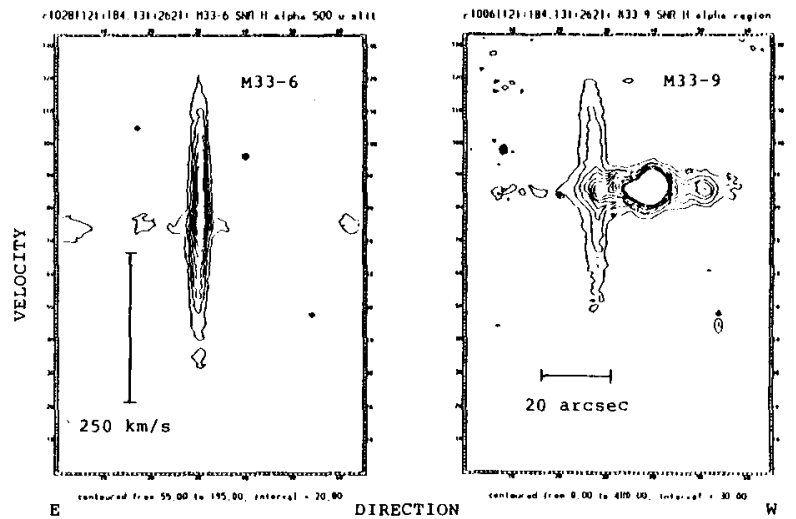

Figure 1: Representative velocity profiles for two M33 remnants. The object on the left shows almost no H II contamination while the object on the right is embedded in the edge of a bright $\mathrm{H}$ II region.
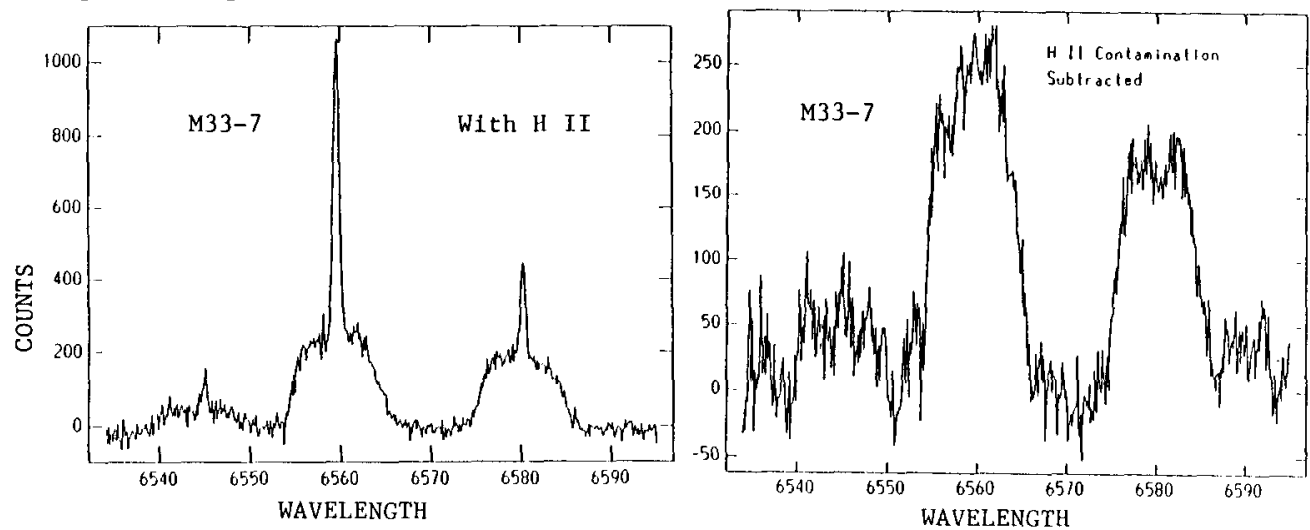

Figure 2: Extracted spectra for SNR M33-7, before and after removal of H Il contamination. Note the square shape of the broad SNR profile.

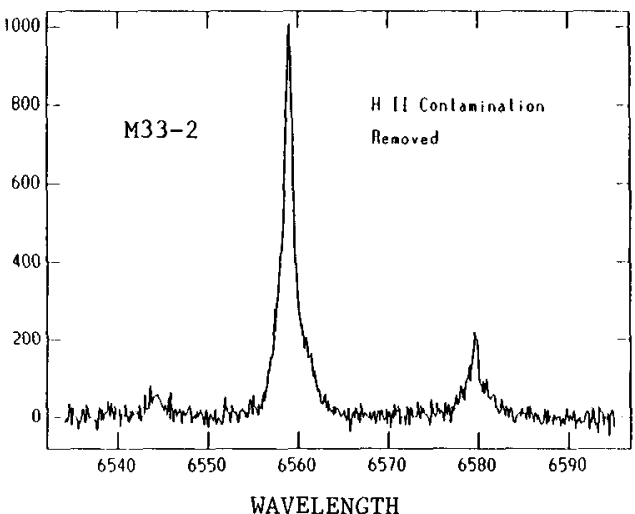

Figure 3: Line profiles for a centrally-peaked SNR, M33-2. Note asymmetry of profile. 
yielding 0.755 arcsec pixels: Vignetting of the long slit limited spatial coverage to about 3 arcmin, but this was much larger than any of the SNRs. The $500 \mu$ slit width corresponded to $\sim 3$ arcsec, and measurement of comparison spectra indicates a velocity resolution of $\sim 25$ $\mathrm{km} \mathrm{s}^{-1}$ was achieved. The slit was wide enough that most if not all of the light from each remnant entered the slit: Thus, integrated velocity profiles were obtained. Seven of the objects were also observed at [O III] $\lambda 5007$ with the same set-up, but at lower signal-to-noise.

The data have been reduced using IRAF at KPNO and at Johns Hopkins. Instrumental curvature was quite apparent in the comparison lines, so the data were rectified so that spatial information was exactly along the lines and the dispersion direction exactly along the columns. Bad columns and cosmic rays were removed from the regions of interest, and a general "background" (including night sky and sometimes diffuse emission from M33) was subtracted. One dimensional spectra corresponding to the SNRs or adjacent H II emission could then be extracted and measured. Representative examples of the two dimensional data and extracted profiles are shown in Figures 1 - 3.

Table 1

Derived Properties of SNRs ${ }^{2}$

\begin{tabular}{lcrrrr}
\hline Object & $\begin{array}{c}\mathrm{V}_{\text {bulk }} \\
\left(\mathrm{km} \mathrm{s}^{-1}\right)\end{array}$ & $\begin{array}{c}\text { FWHM } \\
\left(\mathrm{km} \mathrm{s}^{-1}\right)\end{array}$ & $\begin{array}{c}\text { \% H II } \\
\text { Contam. }\end{array}$ & $\begin{array}{c}\mathrm{D}_{\text {ech }} \\
(\operatorname{arcsec})\end{array}$ & $\begin{array}{c}\text { Size }^{\mathrm{b}} \\
(\operatorname{arcsec})\end{array}$ \\
\hline M33-2 & 263 & 59 & $<1$ & 2.0 & 2.0 \\
M33-4 & 183 & 52 & 12 & 7.7 & 5.1 \\
M33-6 & 345 & 237 & 2 & $<1.0$ & 2.6 \\
M33-7 & 331 & 443 & 21 & 1.7 & $<1.7$ \\
M33-8 & 313 & 151 & 34 & 3.0 & 3.1 \\
M33-9 & 272 & 256 & 25 & 2.7 & 2.0 \\
M33-11 & 185 & 100 & 4 & 3.2 & 2.0 \\
M33-16 & 224 & 91 & 54 & 1.5 & 1.7 \\
M33-18 & 208 & 142 & 37 & 7.3 & 10.9 \\
M33-20 & 103 & 82 & $>70$ & $3.5-5$ & 7.1 \\
\hline
\end{tabular}

${ }^{2}$ Measurements shown pertain to the $\mathrm{H} \alpha$ profile.

${ }^{b}$ From Blair and Kirshner (1985).

\section{Results}

The presence of $\mathrm{H}$ II emission which potentially contaminates the SNR spectra was judged by inspection of CCD imagery of the remnants (Long et al., this conference) as well as the presence of low velocity emission adjacent to or overlapping the high velocity SNR profiles. In addition, the $[\mathrm{N} \mathrm{II}] \lambda 6584 / \mathrm{H} \alpha$ ratio is considerably lower in $\mathrm{M} 33 \mathrm{H}$ II regions than in the SNRs, so the ratio of these lines in the extracted data also provides clues as to whether low velocity emission is photoionized or simply SNR emission with low velocity along the line of sight. Nearly all of the objects we observed showed effects of contamination at some level, ranging from essentially negligible to as much as $70 \%$ of the total flux measured at the SNR position. To the extent possible, the $\mathrm{H}$ II contamination has been subtracted, yielding broad profiles which represent the integrated bulk velocity profiles for each remnant. (This is not strictly true for some of the larger diameter remnants, where a somewhat smaller fraction of the total light was sampled.) The effects of this contamination on the abundance estimates using low resolution spectral data (Blair and Kirshner 1985) have not been assessed, but it will be considerable for several of the objects. A summary of some measured parameters for each remnant is given in Table 1. 
The integrated profiles show a variety of shapes, ranging from almost square (see Figure 2) to centrally peaked with faint, broad wings (see Figure 3). The bulk velocities listed in Table 1 were determined by measuring the full width of the line profile at zero intensity and dividing by two. Some information on the profile shape can be obtained by comparing the FWHM with the bulk velocity of each object: the larger the FWHM, the more "boxy" the profile. Square profiles probably indicate fairly uniform expansion of a thick shell, where the front and back of the shell produce the sides of the box and the material moving in the plane of the sky fills in the center. Centrally peaked profiles with faint, broad wings can be modeled assuming a shock wave passing through a cloudy medium ( $c f$. Norman et al., this conference): shocks are driven into the clouds and accelerate them to a significant fraction of the shock velocity ( $c f$. McKee, Cowie and Ostriker 1978).

This may help explain the apparent discrepancy between the large bulk velocities indicated for the remnants in Table 1 and the need for relatively slow shocks to explain the relative line intensities in (corrected) low resolution spectra. However, bulk motions of 200-300 $\mathrm{km} \mathrm{s}^{-1}$ imply even faster shocks in the intercloud region. These shocks might be unstable (cf. Innes, Giddings and Falle 1987), but would produce intense UV emission. In the two M33 SNRs detected with IUE, neither appeared to be due to a high velocity shock (Blair and Raymond 1984). These objects should also be strong soft X-ray sources, but only one M33 SNR (\#9) corresponds roughly in position with an Einstein source (Long et al. 1981; Markert and Rallis 1983). However, only sources with $\mathrm{L}_{\mathrm{x}} \geq 10^{37} \mathrm{ergs} \mathrm{s}^{-1}$ would have been seen.

We have estimated the diameters of the SNRs from the echelle spectra in the following way: the lines containing the red or blue wing of the high velocity SNR emission were summed and a FWHM measured for the profile across the dispersion. This was then deconvolved from the seeing profile which was measured to be $\sim 2$ arcsec. These diameters, shown as $\mathrm{D}_{\text {ech }}$ in Table 1 , actually refer only to the $\mathrm{E}-\mathrm{W}$ dimension. Our measurements range from considerably smaller to considerably larger than diameters measured directly from images, and cause some concern about the reliability of diameter estimates from images alone.

\section{Future Work}

In addition to comparing the profiles at $[\mathrm{N} \mathrm{II}] \lambda 6584$ and $[\mathrm{O}$ III $] \lambda 5007$ to $\mathrm{H} \alpha$, we need to obtain echelle profiles of these SNRs at $[S$ II] $\lambda \lambda 6717,6731$. Since these lines are density sensitive, we will not only be able to obtain the average SNR density but will also get the density of the $\mathrm{H}$ II component into which many of the remnants appear to be expanding. This will permit a much better understanding of the interaction of each remnant with the surrounding interstellar medium. We also hope to use our integrated line profiles to identify SNRs embedded in giant $\mathrm{H}$ II regions in M33.

\section{References}

Blair, W. P., and Kirshner, R. P. 1985, Ap. J., 289, 582.

Blair, W.P., and Raymond, J.C. 1984, in Six Years of IUE Research, NASA CP-2349, p. 103.

Chu, Y.-H., and Kennicutt, R. C., Jr. 1986, Ap. J., 311, 85.

Cox, D. P., and Raymond, J. C. 1985, Ap. J., 298, 651.

Dopita, M. A., Binette, L., D'Odorico, S., and Benvenuti, P. 1984, Ap. J., 276, 653.

Innes, D. E., Giddings, J. R., and Falle, S. A. E. G. 1987, M.N.R.A.S., 224, 179.

Long, K.S., D'Odorico, S., Charles, P.A., and Dopita, M.A. 1981, Ap. J. (Letters), 246, L61.

Markert, T. H., and Rallis, A. D. 1983, Ap. J., 275, 571.

McKee, C. F., Cowie, L. L., and Ostriker, J. P. 1978, Ap. J. (Letters), 219, L23. 